\title{
NÍVEL DE DEPENDENCIA A NICOTINA E FORÇA MUSCULAR PERIFÉRICA EM TABAGISTAS
}

\section{DEPENDENCE LEVEL ON NICOTINE AND PERIPHERAL MUSCLE STRENGTH IN SMOKERS}

\author{
Luciana Bilitário Macêdo*, Igor Alonso Andrade Oliveira**, Daniele Brito dos Santos**, \\ Francisco Tiago Oliveira de Oliveira***, Cristiane Maria Carvalho Costa Dias****, Aquiles \\ Assunção Camelier*****
}

\begin{abstract}
Autor para correspondência: Luciana Bilitário Macedo - lucianabilitario@bahiana.edu.br
* Mestre em Medicina e Saúde Humana; Professora Assistente da Escola Bahiana de Medicina e Saúde Pública; Docente Universidade do Estado da Bahia, UNEB, Salvador - BA, Brasil

**Discente de Fisioterapia da Escola Bahiana de Medicina e Saúde Pública; Salvador - BA, Brasil

***Especialista em Fisioterapia na Terapia Intensiva Adulto pelo COFFITO/ASSOBRAFIR; Professor Assistente da Escola Bahiana de Medicina e Saúde Pública; Salvador - BA, Brasil

****Doutora em Medicina e Saúde Humana; Professora Adjunta de Fisioterapia da Escola Bahiana de Medicina e Saúde Pública; Salvador - BA, Brasil

*****Professor Adjunto da Escola Bahiana de Medicina e Saúde Pública, Docente Universidade do Estado da Bahia, UNEB, Salvador - BA, Brasil
\end{abstract}

\section{R E S U M O}

\begin{abstract}
Introdução: A nicotina é a principal responsável pela dependência em tabagistas e seus efeitos nos sistemas nervoso e cardiovascular são conhecidos. Não está claro o impacto na força muscular periférica. Objetivo: verificar se existe correlação entre os níveis de dependência a nicotina e a força muscular periférica (FMP). Metodologia: corte transversal com tabagistas admitidos no Programa "Deixando de Fumar sem Mistérios". Após a assinatura do TCLE, foi aplicado o questionário sócio demográfico, tolerância de Fagerström e IPAQ - versão curta. Foi realizado o Handgrip para mensuração da FMP. Utilizado o teste de correlação de Spearman para verificar relações e o teste $T$ de Student para

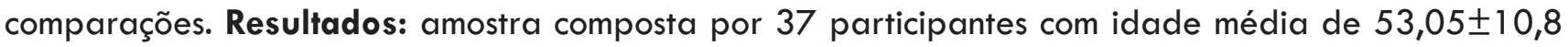
anos quais 26 são mulheres. O nível de dependência a nicotina, $18(48,6 \%)$ indivíduos apresentaram elevada dependência, $17(45,9 \%)$ baixa dependência, e dois $(5,4 \%)$ média dependência. A mediana do escore FTND foi de cinco $(3-6,5)$ e a da carga tabágica foi de $23,4(22,2-50,0)$. A força muscular periférica predita obteve média de 33,68 $\pm 8,64 \mathrm{Kgf}$, enquanto a encontrada obteve mediana de 28,3 (22,9-38,0) Kgf. Não houve diferença entre a força muscular periférica predita e encontrada. Conclusão: houve correlação leve e inversa entre o escore do Fargestrom e a FMP avaliada. Entretanto não foi encontrada correlação entre a carga tabágica e a força muscular periférica (FMP) e diferença entre a FMP predita e encontrada.
\end{abstract}

Palavras Chaves: Hábito de Fumar, Abandono do Uso do Tabaco, Força Muscular e Sistema Musculo Esquelético. 
Introduction: Nicotine is the main responsible for dependence in smokers and yours effects on the nervous and cardiovascular systems are belevied. It is not clear that may interfere with peripheral muscle strength. Objective: To determine whether there is a correlation between the levels of addiction to nicotine and peripheral muscle strength. Methodology: Cross-sectional with admitted smokers in the program "Leaving Smoking without Mysteries." After signing the consent form, applied the demographic questionnaire, tolerance and Fagerstrom, IPAQ - short version. Handgrip was performed to measure the PMS. Spearman correlation test for relation and the Student $t$ test for comparisons was used. Results: Sample of 37 participants with an average age of $53.05 \pm 10.8$ years, 26 are women. The level of addiction to nicotine, $18(48.6 \%)$ subjects have high dependency, $17(45.9 \%)$ low dependence, and two $(5.4 \%)$ average dependency. The median FTND score was five (3 to 6.5) and the smoking history was 23.4 (22.2 to 50.0). Peripheral muscle strength obtained predicted average mean of $33.68 \pm 8.64 \mathrm{kgf}$, while the median obtained was 28.3 (22.9 to 38.0) kgf. There was no difference between peripheral muscle strength predicted and found. Conclusion: There is a slight and inverse correlation between the score of Fargestrom and PMS assessed. However it was not found correlation was found between smoking history and peripheral muscle strength (PMS) and difference between the predicted and found PMS.

Key Words: Smoking Smoking, Abandonment of Tobacco Use, Muscle Strength and Musculoskeletal System. 
O tabagismo é considerado pela Organização Mundial de Saúde (OMS) uma doença crônica e de alta prevalência de consumo'. Estima-se que no ano de 2014, o hábito de fumar foi responsável por mais de seis milhões de morte no mundo ${ }^{2}$. No Brasil são fumados cerca de 110 milhões de unidades de cigarro por ano, o que leva a mais de 200 mil mortes por ano em decorrência do uso excessivo do cigarro 3,4 . Devido a isto, o Ministério da Saúde do Brasil, desenvolveu o programa Deixando de Fumar sem Mistérios, com o objetivo de auxiliar a cessação tabágica e reduzir os custos para o Sistema Único de Saúde ${ }^{4}$.

Dentre os produtos mais utilizados pelos tabagistas encontra-se o cigarro, devido ao seu baixo custo e fácil acesso ${ }^{3}$. Nele encontramos por volta de 4.700 substâncias, dentre elas a nicotina, principal responsável pela dependência ${ }^{5}$. Estudos apontam suas consequências sistêmicas, podendo ocorrer impacto na função muscular. Essa afirmação é fundamentada na ação da nicotina no sistema nervoso, como a liberação de acetilcolina na placa motora, além de ligar-se aos seus receptores, o que diminui a fase de relaxamento da musculatura, aumentando a probabilidade de lesão do tecido ${ }^{6}$. Além disso, a nicotina reduz a capacidade da proliferação de miofibroblastos, responsáveis pela regeneração dos tecidos musculares ${ }^{7}$. Por estes motivos acreditamos que estes fatores associados possam influenciar na força muscular periférica (FMP) dos tabagistas.

A força muscular é a capacidade do músculo em contrair $^{8}$ sendo um importante preditor de mortalidade ao longo dos anos?. A sua diminuição gera impacto na qualidade de vida, a capacidade funcional e das atividades de vida diária (AVDs) ${ }^{10}$ devido a restrições físicas trazidas pela fraqueza muscular ${ }^{11}$. Sabe-se que algumas doenças frequentes em tabagistas ${ }^{12}$ como a doença pulmonar obstrutiva crônica (DPOC) tem influência na FMP. Entretanto pouco se sabe sobre o comportamento da FMP em tabagistas que não têm o diagnóstico da doença pulmonar obstrutiva crônica.

O presente estudo teve como objetivo verificar se existe correlação entre os níveis de dependência a nicotina e a força muscular periférica de tabagistas.

\section{MATERIAIS E MÉTODOS}

Trata-se de um estudo observacional do tipo corte transversal em tabagistas admitidos no programa "Deixando de Fumar Sem Mistérios" do Ministério da Saúde do Brasil, que acontece na Clínica Escola Avançada de Fisioterapia da Escola Bahiana de Medicina e Saúde Pública em Salvador - BA, no período de dezembro de 2015 a julho de 2016. Aprovado pelo comitê de ética e pesquisa com CAAE: 246229815.7.0000.5029. Foram incluídos: tabagistas no momento da admissão no programa, de ambos os sexos, com idade igual ou superior a 18 anos e excluídos aqueles que apresentassem dificuldade para compreensão dos questionários aplicados, diagnóstico de DPOC e (ou) limitações físicas para realização do teste de força muscular periférica.

Todos os indivíduos assinaram o do Termo de Consentimento Livre e Esclarecido (TCLE) e foram encaminhados a uma sala reservada para aplicação dos questionários. Para mensuração do nível de dependência a nicotina utilizou-se ○ "Questionário de Tolerância de Fargestrom", um instrumento que pontua as atividades relacionadas ao hábito de fumar. Após a somatória dos pontos gera-se um escore que permite classificar a dependência em: baixa, moderada e elevada. Quanto maior a pontuação, maior será $\circ$ nível de dependência a nicotina $^{13}$.

Para verificação do nível de atividade física, foi aplicado o "Questionário Internacional de Atividade Física" - IPAQ Versão Curta, que leva em consideração o tempo que $\circ$ indivíduo gasta realizando atividades vigorosas ou moderadas, e que $\circ$ mesmo permanece sentado ou deitado durante $\circ$ dia. Este questionário permite classificalos em: sedentário, moderadamente ativo e ativo ${ }^{14}$. Para mensuração da força muscular periférica, utilizou-se o Handgrip (força de preensão palmar), 
com ○ dinamômetro da marca Camry, modelo EH 101 digital, de acordo com o protocolo da Sociedade Americana de Terapeutas da Mão (ASTH), o membro testado foi o dominante, com três contrações de 30 segundos e intervalos de um minuto entre as mensurações. $O$ valor adotado para posterior análise foi o maior encontrado nas três repetições ${ }^{15}$. Os valores de referência foram estabelecidos através da formula de Novaes 2009: $F M P-D=39,996(0,382 x i d a d e)+(0,174$ xpeso $)$ $+(13,628 \times$ xênero*) e FMP $-E=44,968$ $(0,42$ xidade $)+(0,110$ xpeso $)+(9,274$ xgênero* $)$ Gênero* (Homens $=1$, Mulheres $=0)^{16}$.

O tamanho amostral foi estimado através da calculadora Stats ToDo (https://www.statstodo.com/ index.php), com o comando de correlação entre os níveis de dependência a nicotina e a força muscular periférica, o coeficiente de correlação adotado foi de 0,4 , para uma moderada correlação poder do teste $80 \%$ com um nível de significância de $5 \%$, estimando uma população de 37 participantes. $O$ Software Statistical Package for Social Sciences (SPSS) versão 14.0 para windows foi utilizado para elaboração e análise dos dados através da estatistica descritiva e analitica. A normalidade das variáveis foi verificada através da análise estatística descritiva e o teste Kolmogorov-Smirnov, sendo a descritiva considerada como soberana, em casos de discordância.

O coeficiente de correlação de Spearman foi utilizado para verificar a correlação entre o escore do FTND e a força muscular periférica, assim como a carga tabágica com a força muscular periférica. $O$ teste $T$ de Student pareado foi utilizado para comparar a força muscular periférica predita com a encontrada.

\section{RESULTADOS}

Amostra inicial composta por 39 participantes nos quais dois foram excluídos, um por dificuldade de compreensão dos questionários aplicados e um por limitação na aplicação do teste de força muscular periférica, totalizando uma amostra de 37 indivíduos. Destes $26(70,3 \%)$ são do sexo feminino, com idade média de 53,05 $\pm 10,8$ anos e IMC de $25,8 \pm 4,4 \mathrm{~kg} / \mathrm{m} 2$. Conforme descrito na Tabela 1 .

Tabela 1. Características sociodemográficas e antropométricas dos participantes do programa "Deixando de Fumar sem Mistérios". Salvador - Bahia. $\mathrm{N}=37$

\begin{tabular}{lll}
\hline Variáveis & $\mathbf{n}$ & $\%$ \\
\hline Sexo & 11 & 29,7 \\
Masculino & 26 & 70,3 \\
Feminino & & \\
Estado Civil & 16 & 43,2 \\
Solteiro & 13 & 35,1 \\
Divorciado & 5 & 13,5 \\
Casado & 3 & 8,1 \\
Viúvos & & \\
Escolaridade & 1 & 2,7 \\
Analfabeto & 6 & 16,3 \\
$1^{\circ}$ Grau & 17 & 51,3 \\
$2^{\circ}$ Grau & 11 & 29,7 \\
Superior & & \\
Renda Familiar & 15 & 40,5 \\
Até 2 salários & 12 & 32,4 \\
Entre 2 e 4 salários & 7 & 18,9 \\
Entre 4 e 10 salários & 7 & 2,7 \\
Entre 10 e 20 salários & 1 & 5,4 \\
Não declarado & 2 & DP \\
Variável & Média & 10,8 \\
Idade (anos) & 53,05 & 4,4 \\
IMC & 25,8 & \\
IMC = Indice de Massa Corporal, DP = Desvio Padrão &
\end{tabular}

Quanto ao auto relato de doenças associadas ao tabagismo, $22(59,5 \%)$ participantes afirmaram possuir, sendo mais frequente a hipertensão arterial sistêmica $14(37,8 \%)$, seguidas por doenças respiratórias com três $(8,1 \%)$, diabetes mellitus dois $(5,4 \%)$ e doenças vasculares centrais um $(2,7 \%)$. De acordo com o IPAQ 17 (46\%) participantes foram considerados ativos, dez (27\%) moderadamente ativo e dez $(27 \%)$ sedentários.

Quando analisado o nível de dependência a nicotina, $18(48,6 \%)$ indivíduos apresentaram elevada dependência, 17 (45,9\%) baixa dependência, e dois $(5,4 \%)$ média dependência. A mediana do escore do questionário de tolerância de Fargestrom foi de cinco $(3-6,5)$ e a da carga tabágica foi de 
$23,4(22,2-50,0)$, conforme descrito na tabela 2.

Tabela 2. Doenças associadas, nível de atividade física e nível de dependência a nicotina dos participantes do programa "Deixando de Fumar sem Mistérios". Salvador - Bahia. $\mathrm{N}=37$.

\begin{tabular}{lcc}
\hline \multicolumn{1}{c}{ Variáveis } & Mediana & Quartil \\
\hline Escore do FTND & 5 & $3-6,5$ \\
Carga Tabágica & 23,4 & $22,2-50$ \\
\hline \multicolumn{1}{c}{ Variáveis } & $\mathbf{n}$ & $\%$ \\
\hline
\end{tabular}

\section{Doenças Associadas}

\begin{tabular}{lcc} 
Não possuem & 15 & 40,5 \\
HAS & 14 & 37,8 \\
Doenças Respiratórias & 3 & 8,1 \\
Díabetes Melitus & 2 & 5,4 \\
Outros & 2 & 5,4 \\
DVC & 1 & 2,7 \\
Nível de atividade Física & & \\
Sedentário & 10 & 27,0 \\
Moderadamente Ativo & 10 & 27,0 \\
Ativo & 17 & 46,0 \\
Nível de Dependência & & \\
Baĩxa & 17 & 45,9 \\
Moderada & 2 & 5,4 \\
Elevada & 18 & 48,6 \\
\hline
\end{tabular}

FTND = Questionário de Tolerância de Fargestrom, HAS = Hipertensão Arteria Sistemica, DR = Doenças Respiratórias, DM = Diabetes Mellitus, DVC = Doença Vascular Central.
A força muscular periférica predita obteve média de $33,68 \pm 8,64 \mathrm{Kgf}$, enquanto a encontrada $31,32 \pm 11,4$. Não houve diferença estatística entre a força muscular periférica predita e encontrada. Conforme descrito na tabela 3.

Tabela 3. Força muscular periférica predita e encontrada por nível de dependência a nicotina dos participantes do programa "Deixando de Fumar sem Mistérios". Salvador - Bahia. $\mathrm{N}=37$.

\begin{tabular}{lccc}
\hline Níveis de Dependência & FMP - Prediła & FMP - Encontrada & P \\
\hline Baixa & $35,7 \pm 10,04$ & $34,1 \pm 11,9$ & 0,642 \\
Moderada & $38,3 \pm 9,73$ & $29,1 \pm 8,6$ & 0,054 \\
Elevada & $31,2 \pm 6,6$ & $28,8 \pm 11,1$ & 0,352 \\
Geral & $33,6 \pm 8,6$ & $31,32 \pm 11,4$ & 0,221 \\
\hline Teste T de Student pareado, FMP = Força Muscular Periférica &
\end{tabular}

Não foi encontrada uma correlação entre a carga tabágica e a força muscular periférica ( $p=$ $0,21)$. Entretanto, observou-se uma leve e inversa correlação $(p=0,02$ e $r=-0,366)$ entre a força muscular periférica com o escore FTND.

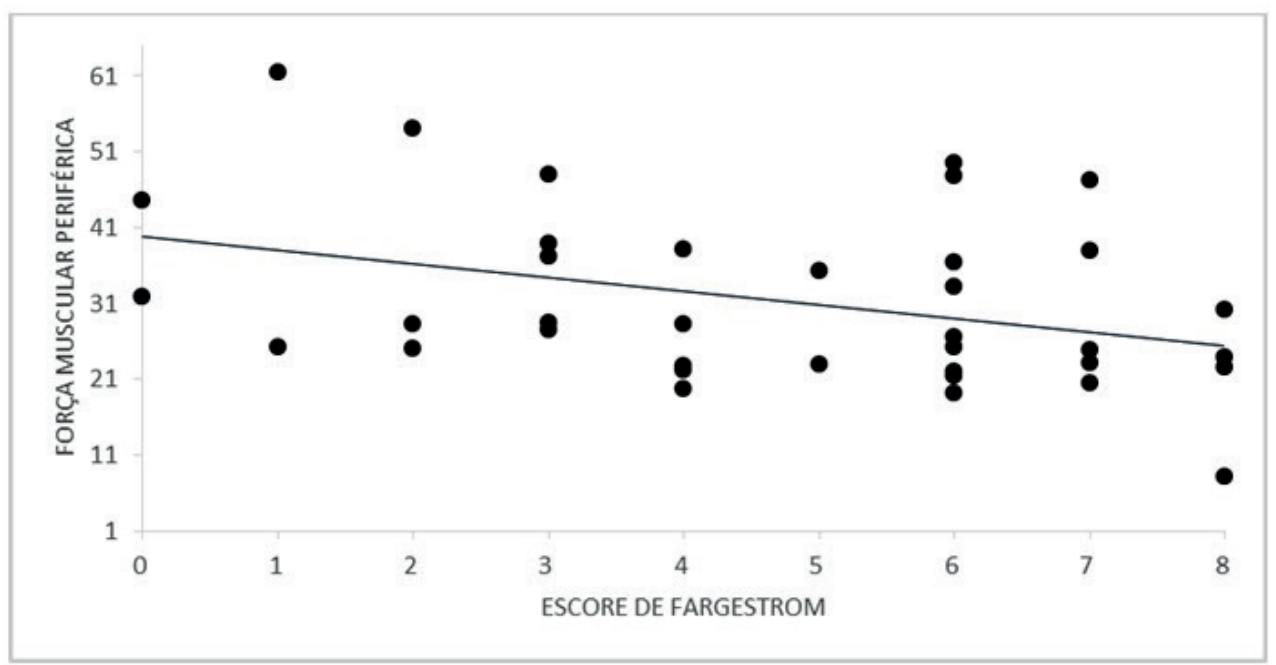

Teste de correlação de Spearman, $p=0,02 r=-0,366$

Gráfico 1. Correlação entre o escore do FTND e a força muscular periférica encontrada dos participantes do programa "Deixando de Fumar sem Mistérios". Salvador - Bahia. N=37. 


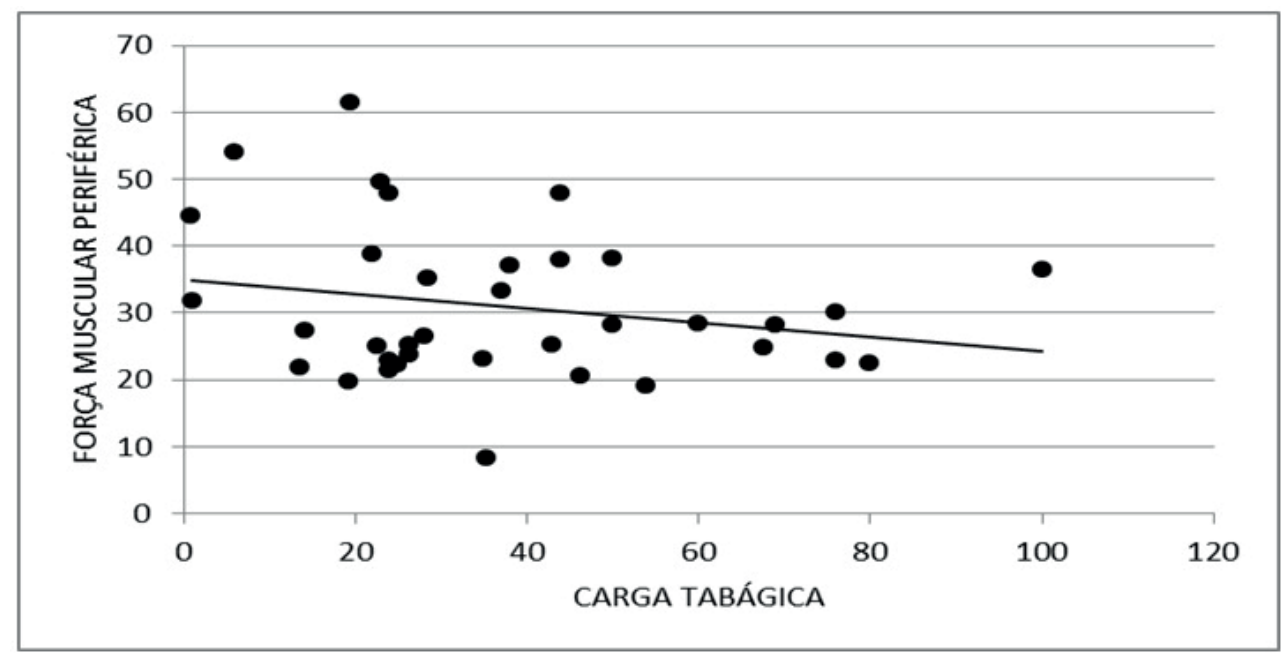

Teste de correlação de Spearman, $p=0,21$

Gráfico 2. Correlação entre a carga tabágica e a força muscular periférica encontrada dos participantes do programa "Deixando de Fumar sem Mistérios". Salvador - Bahia. N=37.

\section{DISCUSSÃO}

A dependência da nicotina é um processo complexo que envolve a ação farmacológica, comportamental e psicológica do individuo ${ }^{13}$. Esta é associada ao número de cigarros fumados por dia, tempo de fumo, predisposição genética e condições socioambientais ${ }^{14}$. Na amostra estudada foi encontrada uma correlação leve e inversa entre os níveis de dependência de nicotina e a força muscular periférica dos tabagistas. Entretanto, apesar desse achado, os valores de FMP predita foram semelhantes ao da força muscular encontrada nos tabagistas avaliados.

Em 2002 Tanamaki ${ }^{17}$ avaliou o comportamento das fibras do músculo sóleo de ratos expostos a fumaça de cigarro e observou que a exposição contribui para declínio da capacidade oxidativa das fibras do tipo I, endurance, devido à redução do aporte de oxigênio ofertado ao musculo. No presente estudo foi verificada através do teste de preensão palmar a força isométrica máxima do individuo ${ }^{18}$ que avalia as fibras do tipo II, com característica de contração rápida. O estudo de Dourado $2006^{19}$ revelou que existem alterações de fibras do tipo I nos membros superiores de pessoas com DPOC, doença decorrente do tabagismo. Outro estudo revelou que as fibras do tipo II dos membros superiores, não sofrem grandes alterações devido à sua utilização na maioria das $\mathrm{AVDs}^{18}$. Esses resultados corroboram com os nossos, principalmente quando associado a ausência do diagnóstico de DPOC.

A força muscular periférica é um importante preditor da capacidade funcional e de mortalidade ao longo dos anos ${ }^{18,19}$. Além disso, o estudo de Ichinose et $\mathrm{al}^{.}{ }^{20} \mathrm{em} 2010$ revelou que existe uma redução na produção de oxido nítrico e aumento na síntese de colágeno em ratos expostos a fumaça, fatores que podem contribuir para lesões de tendões musculares devido ao aumento da sua elasticidade ${ }^{20}$. Por este motivo, se faz necessária a mensuração da FMP nesta população, para guiar os profissionais de saúde quanto as suas práticas clinicas. $O$ handgrip é um teste descrito, validado e seguro para a sua mensuração, podendo ser facilmente utilizado devido ao seu baixo custo e fácil aplicação ${ }^{18,21}$.

A amostra deste estudo foi composta principalmente por mulheres. Segundo o estudo de Gomes em 2007 as mulheres procuram mais os serviços de saúde por questões históricas e culturais ${ }^{22}$. Associado $a$ isso a idade média foi de 53,05 anos, período em que as mulheres saem da menopausa, fase marcada por diversas alterações fisiológicas e psicológicas ${ }^{23}$.

Estas alterações diminuem $\circ$ seu metabolismo 
energético em repouso, aumentam a tendência para o desenvolvimento de câncer e a presença de sarcopenia tendo por consequência a redução da FMP $^{23,24}$.

Em relação às doenças associadas ao tabagismo destacam-se: o câncer, as doenças cardiovasculares e respiratórias como as principais causas de internação ${ }^{3}$. No presente estudo observamos uma predominância da hipertensão arterial, provavelmente esse achado é devido ao auto relato dos participantes do programa que gera consequentemente um sub diagnóstico das doenças associadas ao tabagismo.

Este estudo apresentou como limitação ter utilizado apenas um teste para avaliar força muscular, além de não desenvolver um grupo controle com indivíduos não tabagistas para comparação das variáveis dependentes.

\section{CONCLUSÃO}

Na população estudada houve correlação leve e inversa entre o escore do Fargestrom e a FMP avaliada. Entretanto a força muscular periférica (FMP) encontrada é semelhante a força muscular periférica predita.

\section{AGRADECIMENTOS}

Este artigo é parte da tese de doutorado de Luciana Bilitário Macedo no Programa de Pós Graduação Stricto Sensu em Medicina e Saúde Humana da Escola Bahiana de Medicina e Saúde Pública.

Projeto financiado pela Fundação de Amparo à Pesquisa do Estado da Bahia, FAPESB, através de bolsa de iniciação científica aos acadêmicos.

\section{REFERÊNCIAS}

1. World Health Organization. Global health risks. WHO Libr. Cat. Data Glob. 2009

2. World Health Organization. Global progress report. WHO Libr. Cat. 2014

3. Pinto M, Uga MAD. Os custos de doenças tabaco-relacionadas para o Sistema Único de Saúde. Cad. Saúde Pública. 2010;26(6):1234-1245. doi: 10.1590/S0102$311 \times 2010000600016$

4. Instituto Nacional do Câncer. Observatório da Política Nacional de Controle do Tabaco: Doenças Relacionadas ao Tabagismo [Internet]. 2016.

Disponível em: http://www2.inca.gov.br/wps/wcm/ connect/observatorio_controle_tabaco/site/home/ dados_numeros/prevalencia-de-tabagismo

5. Rose JE, Behm FM, Westman EC, Mathew RJ, London ED, Hawk TG. PET Studies of the Influences of Nicotine on Neural Systems in Cigarette Smokers. J. Pysychiatry. 2003; 160(2)323-333. doi: 10.1176/appi.ajp.160.2.323

6. Cruz FC, Planeta CS. Bases neurofisiológicas da dependência do tabaco. Rev. Psiquiatr. Clin. 2005;32(12):251-258. doi: 10.1590/S0101 60832005000500002

7. Campos ACL, Alves MR, loshii SO, MoraesJunior H, Sakamoto D, Gortz LW. Influência da nicotina na proliferação de miofibroblastos e de vasos sanguíneos no tecido cicatricial da parede abdominal de ratos lactentes: estudo imunoistoquímico. $A B C D$. Arq. Bras. Cir. Dig. 2010;23(4):222-227. doi: 10.1590/S010267202010000400003

8. Dourado V, Antunes L, Tani S, Filho V, Cunha $M$, Godoy I. Correlação entre força de preensão palmar e força muscular periférica em pacientes com doença pulmonar obstrutiva crônica. Rev. Bras. Fisioter. 2004;8(Sup):109-1 10

9. Pietrobon RC, Barbisan JN, Manfroi WC. Use of the Fagerström Test for Nicotine Dependence As an Instrument To Measure Nicotine Dependence. Rev 
HCPA. 2007;27(3):31-36

10. Lima EC, Macedo LB. Nível de Atividade Física e Tabagismo em Moradores de uma Comunidade em Salvador. Rev Pesqui. em Fisioter. 2012;2(1):25-31. doi: 10.17267/2238-2704rpf. v2i 1.83

11. Reis MM, Arantes PMM. Medida da força de preensão manual- validade e confiabilidade do dinamômetro saehan. Fisioter. e Pesqui. $2011 ; 18(2): 176-181$

12. Fernandes $A A$, Silva $C D$, Vieira $B C$, Marins JCB. Validade preditiva de equações de referência para força de preensão manual em homens brasileiros de meia idade e idosos. Fisioter. e Pesqui. 2012;19(4):351-356. doi: 10.1590/ S1809-29502012000400010

13. França SAS, Neves ALF, Souza TAS, Martins NCN, Carneiro SR, Sarges ESNF et al. Factors associated with smoking cessation. Rev. Saude Pública. 2015;49:10. doi: doi:10.1590/S00348910.2015049004946

14. Rosemberg J, Rosemberg AMA, Moraes MA. Nicotina: Droga Universal. Secr. Saude; Cent. Vigilância Epidemiológica. 2003. P. 178.

15. Nakatani T, Nakashima T, Kita T, Ishihara A. Responses of Exposure to Cigarette Smoke at Three Dosage Levels on Soleus Muscle Fibers in WistarKyoto and Spontaneously Hypertensive Rats. J. Pharmacol. 2002;90:157-163. doi: 10.1254/ iip.90.157

16. Dias JA, Ovando AC, Külkamp W, Junior NGB. Força de preensão palmar: métodos de avaliação e fatores que influenciam a medida. Rev Bras Cineantropom desempenho Hum. 2010;12(3):209216. doi: $10.5007 / 1980-0037.2010 v 12$ n3p209

17. Dourado VZ, Tanni SE, Vale AS, Faganello MM, Sanchez FF, Godoy I. Manifestações Sistêmicas na Doença Pulmonar Obstrutiva Crônica. J. Bras. Pneumol. 2006;32(2):161-171. doi: 10.1590/ S1806-37132006000200012

18. Lauretani $F$, Russo $C R$, Bandinelli $S$, Bartali $B$, Cavazzini $C$, Di lorio A et al. Age-associated changes in skeletal muscles and their effect on mobility : an operational diagnosis of sarcopenia. J Appl Physiol. 2003;95(5):1851-1860. doi: 10.1152/japplphysiol.00246.2003

19. A Soares. Correlação entre os testes de Dinamometria de Preensão Manual, Escapular e Lombar. Rev. Acta Bras. do Mov. Hum. $2012 ; 2(1): 65-72$

20. Ichinose R, Sano H, Kishimoto KN, Sakamoto $N$, Sato $M$, Itoi E. Alteration of the material properties of the normal supraspinatus tendon by nicotine treatment in a rat model. Acta Orthop. 2010;81(5):634-8. doi: $10.3109 / 17453674.2010 .524595$

21. Gale CR, Martyn CN, Cooper C, Sayer AA. Grip strength , body composition , and mortality. 2007;36(1):228-235. doi: 10.1093/ije/dyl224

22. Gomes R, Nascimento EF, Araujo FC. Por que os homens buscam menos os serviços de saúde do que as mulheres?. Cad. Saúde Pública. 2007;23(3):565-574. doi: 10.1590/s0102$311 \times 2007000300015$

23. Bonganha V, Santos CF, Rocha J, ChaconMikahil MPT, Madruga VA. Força muscular e composição corporal de mulheres na pós-menopausa: Efeitos do treinamento concorrente. Rev. Bras. Atividade Física e Saúde. 2009;13(2):102-109. 10.12820/ RBAFS.V.13N2P102-109

24. Trevisan MC, Burini RC. Metabolismo de repouso de mulheres pós-menopausadas submetidas a programa de treinamento com pesos (hipertrofia). Rev. Bras. Med. do Esporte. 2007;13(2):133-137. doi: 10.1590/S151786922007000200013 